\title{
Risk factors for delayed healing at the free anterolateral thigh flap donor site
}

\author{
Yoshiro Abe, Keisuke Kashiwagi, Soushi Ishida, Kazuhide Mineda, Yutaro Yamashita, \\ Ichiro Hashimoto \\ Department of Plastic and Reconstructive Surgery, Tokushima University Graduate School of Medical Science, Tokushima, Japan
}

Background The free anterolateral thigh (ALT) flap has been widely used for various kinds of reconstructions. However, delayed healing at the donor site occasionally occurs due to wound dehiscence or the partial loss of grafted skin at the donor site. The aim of the present study was to identify reliable predictive factors for delayed healing at the donor site after the harvest of a free ALT flap.

Methods This study included 52 patients who underwent reconstructive procedures using free ALT flaps. The delayed healing group included patients with wounds at the donor site that had not healed over 3 weeks after surgery, and the normal healing group included patients who showed wound healing within 3 weeks after surgery. Multivariate logistic regression models were created to identify the risk factors for delayed healing at the ALT flap donor site.

Results Among the 52 patients, 24 (46.2\%) showed delayed healing at the donor site, and 6 patients required additional operative treatment. A high preoperative body mass index (BMI), smoking, and skin grafting were found to be significantly associated with delayed healing at the ALT donor site. Of the 37 patients who underwent skin grafting, 23 (62\%) experienced delayed healing at the donor site.

Conclusions A high preoperative BMI, smoking, and skin grafting were risk factors for delayed healing at the free ALT donor site. Skin grafting at the ALT donor site should be avoided in patients with a high BMI or a habit of smoking.

Keywords Reconstructive surgical procedures / Free tissue flaps / Wound healing / Risk factors
Correspondence: Yoshiro Abe Department of Plastic and

Reconstructive Surgery, Tokushima University Graduate School of Medical

Science, 3-18-15 Kuramoto,

Tokushima 770-8503, Japan

Tel: +81-88-633-7296

Fax: +81-88-633-7297

E-mail: y.abe@tokushima-u.ac.jp

Received: 3 Apr 2017 • Revised: 4 Aug 2017 • Accepted: 26 Sep 2017

pISSN: 2234-6163・ elSSN: 2234-6171・https://doi.org/10.5999/aps.2017.00563・ Arch Plast Surg 2018;45:51-57

\section{INTRODUCTION}

The free anterolateral thigh (ALT) flap has become very popular among reconstructive surgeons due to its versatility and utility for various kinds of reconstructions at sites throughout the human body, such as the head and neck, extremities, and trunk [1-4]. The versatility of this type of flap allows the elevation of a cutaneous, fasciocutaneous, or myocutaneous flap. For reconstruction of large defects in soft tissues, an ideal soft-tissue free flap should have the following characteristics: superior vascular supply to the skin, versatility in flap design, a long pedicle, consistent vascular anatomy for easy and safe flap elevation, feasibility of a 2-team approach, and minimal donor site morbidity. The ALT flap has been recognized to have most of these charac- 
teristics. In cases of large defects, this flap can be used with a segment of the vastus lateralis muscle (VLM).

With respect to the ALT flap, surgeons have been most concerned about the vascular anatomy $[5,6]$, the size of the harvested flap area, and its clinical applications [4]. Some studies have reported donor site morbidity related to gait disturbance or lower extremity weakness after the harvest of free ALT fasciocutaneous and myocutaneous flaps [5,7-11]; however, few studies have assessed delayed healing at the donor site because of wound dehiscence associated with primary closure or the partial loss of grafted skin.

Even if skin grafting is performed for a large skin defect at the donor site, epithelialization usually occurs 3 weeks after the operation. Wound dehiscence or the partial loss of grafted skin occasionally results in delayed wound healing at the donor site. If delayed healing occurs, additional treatment is sometimes necessary to close the wound after sufficient wound bed preparation, and the patient is required to stay in the hospital for an extended period. No published reports have examined which factors among patient characteristics and operative techniques, such as the size of the harvested ALT flap, the distribution of the perforators included within the flap, and whether or not the VLM is incorporated, are associated with delayed healing at the donor site after the harvest of a free ALT flap. The aim of the present study was to identify reliable predictive factors for delayed healing at the donor site after the harvest of a free ALT flap.

\section{METHODS}

This study was approved by the Research Ethics Board at Tokushima University and informed consent was obtained.

This study was approved by the Research Ethics Board at Tokushima University (IRB no. 2601) and informed consent was obtained.

\section{Patients}

This study included 52 patients who underwent reconstructive procedures using free ALT flaps at our institution. The lesions that required free ALT flap reconstruction and the recipient sites are presented in Table 1. The main reason for the operation was defect reconstruction after ablation of a malignant tumor in 34 patients (65.4\%), and the most frequent site of free ALT flap reconstruction was the head and neck region $(80.8 \%)$. The study also included patients in whom a portion of the VLM was harvested with the ALT flap.

\section{Operative procedure}

The ALT flap was harvested using standard operative tech- niques $[1,4]$. After making a longitudinal or slightly curved incision under the deep fascia at the anterior aspect of the thigh, the pedicle of the flap was dissected and isolated. If the perforator traveled through the VLM (a musculocutaneous pattern of perforators), intramuscular dissection was performed along the perforator, using a careful and subtle technique with optical magnification (Fig. 1A). When proximal dissection and isolation of the descending branch of the lateral circumflex femoral artery were required, the rectus femoris muscle was detached entirely from the intermuscular connective tissues attached to the vastus intermedius muscle. If the VLM was incorporated into the ALT flap, the muscle nourished by the muscular branch from the descending branch of the lateral circumflex femoral artery was cut and harvested (Fig. 1B).

After dissection of the pedicle, the flap was designed and elevated with some sacrifice of the underlying fascia lata. The defect of the thigh was closed with 3-0 Vicryl (Ethicon Inc., Somerville, NJ, USA) to adapt the dissected VLM with the remaining muscle or the divided rectus femoris muscle. A suction drain was inserted between the rectus femoris muscle and vastus intermedius muscle, although intramuscular dissection was not required. In cases of primary closure, intradermal suturing was performed with 4-0 PDS II (Ethicon Inc.) and dermal suturing was performed with 4-0 or 5-0 black nylon sutures (Keisei Medical Industrial Co., Tokyo, Japan). If necessary, an additional suction drain was inserted beneath the undermined skin. Split-thickness skin grafting was performed using a conventional bolster dressing with or without a mesh procedure if primary closure could not be achieved due to tightness at the donor site (Fig. 1C).

\section{Postoperative donor site wound management and assessment}

For external fixation, a molded plastic splint from the thigh to the lower leg was applied for a week not only in the patients who

Table 1. The lesions that underwent free anterolateral thigh flap reconstruction and the recipient sites

\begin{tabular}{|lc|}
\hline Lesion/Recipient site & No. (\%) \\
\hline Lesion & \\
Malignant tumor & $34(65.4)$ \\
Contracture & $9(17.3)$ \\
Refractory ulcer & $7(13.5)$ \\
Benign tumor & $2(3.8)$ \\
Recipient site & \\
Head and neck & $42(80.8)$ \\
Lower limb & $5(9.6)$ \\
Upper limb & $4(7.7)$ \\
Trunk & $1(1.9)$ \\
\hline
\end{tabular}




\section{Fig. 1. ALT flap harvest and donor site closure}

(A) An intramuscular dissection was performed along the perforator when a perforator traveled through the vastus lateralis muscle. (B) Two segments of the vastus lateralis muscle nourished by the muscular branch from the descending branch of the lateral circumflex femoral artery were incorporated into the ALT skin flap. (C) A split-thickness meshed skin graft with a bolster dressing was applied on the remaining muscles after harvesting the flap. ALT, anterolateral thigh.

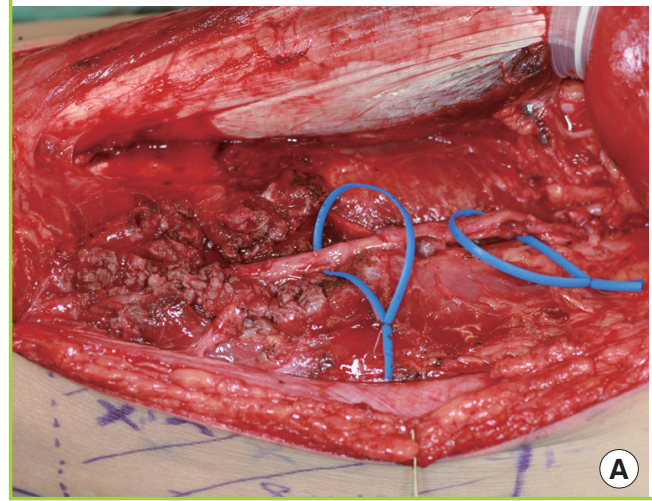

underwent skin grafting, but also those who underwent primary closure, in order to maintain a firm resting position and to reduce the risk of postoperative hematoma or seroma formation. In patients who underwent primary closure, the stitches were removed 7-10 days after the operation. In patients who underwent skin grafting, a bolster dressing was applied for 5-7 days. After removal of the dressing, a hydrophilic ointment was applied every day or every 2 days, depending on the amount of wound discharge.

\section{Statistical analysis}

We divided the patients into a delayed healing group and a normal healing group. The delayed healing group included patients with wounds at the donor site that had not healed over 3 weeks after surgery because of wound dehiscence or the partial loss of grafted skin, and the normal healing group included patients who showed wound healing within 3 weeks after surgery. Multivariate logistic regression models were created to estimate the risk factors for delayed healing at the ALT flap donor site. Variables were included in the models based on existing knowledge of the risk factors for delayed wound healing. We collected the following data from patient records: age; history of hypertension (HT), hyperlipidemia (HL), and diabetes mellitus (DM); habit of smoking; preoperative body mass index (BMI); levels of hemoglobin $(\mathrm{Hb})$, total protein $(\mathrm{TP})$, and albumin. Procedures such as intramuscular dissection for the isolation of perforators or incorporation of the VLM when harvesting a free ALT flap were identified as potentially being associated with delayed wound healing. Therefore, we recorded the following operative factors: size of the harvested ALT and VLM flaps; number of included perforators (single or multiple); perforator type (musculocutaneous or septocutaneous perforator); and closure method (direct closure or skin graft). The laboratory data were collected within 2 weeks before surgery. The size of the harvested ALT flap corresponded to the defect size of the donor site, which was the same size as the applied skin graft. The data were first analyzed using univariate logistic regression analysis. Then, variables with P-values $<0.1$ in the univariate logistic regression analysis were considered for inclusion in the multivariate logistic regression model. All statistical analyses were performed using the statistical software R ver. 2.15.0 (http://www.r-project. org/). P-values $<0.05$ were considered to indicate statistical significance.

\section{RESULTS}

The patient characteristics are presented in Table 2. Of the 52 patients, 24 (46.2\%) showed delayed healing at the donor site (delayed healing group) and 28 (53.8\%) showed normal healing (normal healing group). Of the 24 patients in the delayed healing group, 6 (25\%) required additional operative treatment (Fig. 2 ) and the remaining 18 (75\%) were managed conservatively.

Age; history of HT, HL, and DM; levels of TP and albumin; the incorporated VLM flap size; the multiple perforators; and the musculocutaneous perforator were not found to be associated with delayed healing in the univariate logistic regression analysis. Preoperative BMI, smoking, and skin grafting were found to be significantly associated with delayed healing of the ALT donor site in the multivariate logistic regression analysis after controlling for $\mathrm{Hb}$ levels and the skin flap size (Table 3). The mean preoperative $\mathrm{BMI}$ in the delayed healing group was higher than that in the normal healing group. In multivariate logistic regression analysis, the possibility of a high preoperative BMI was greater in the delayed healing group than in the normal healing 
Table 2. Characteristics of the patients who underwent free ALT flap reconstruction

\begin{tabular}{|c|c|c|}
\hline Characteristic & Delayed healing group $(n=24)$ & Non-delayed healing group $(n=28)$ \\
\hline Age (yr) & $52.8 \pm 15.9(18-80)$ & $59.2 \pm 13.5(29-82)$ \\
\hline Body mass index $\left(\mathrm{kg} / \mathrm{m}^{2}\right)$ & $26.2 \pm 3.3(20.4-34.6)$ & $22.3 \pm 3.4(16-30.1)$ \\
\hline Hypertension (\%) & $5(20.8)$ & $7(25.0)$ \\
\hline Hyperlipidemia (\%) & $8(33.3)$ & $9(32.1)$ \\
\hline Diabetes mellitus (\%) & $3(12.5)$ & $2(7.1)$ \\
\hline Smoking (\%) & 19 (79.2) & $11(39.3)$ \\
\hline Hemoglobin (g/dL) & $13.4 \pm 1.8(10.9-17.2)$ & $12.3 \pm 1.9(7.4-15.6)$ \\
\hline Total protein (g/dL) & $6.9 \pm 0.6(5.6-7.7)$ & $7.0 \pm 0.7(5.6-8.1)$ \\
\hline Albumin (g/dL) & $3.8 \pm 0.4(2.8-4.6)$ & $3.7 \pm 0.5(2.6-4.6)$ \\
\hline Skin flap size $\left(\mathrm{cm}^{2}\right)$ & $171.2 \pm 91.7(59-425)$ & $123.3 \pm 78.0(30-368)$ \\
\hline VLM flap size $\left(\mathrm{cm}^{2}\right)$ & $32.8 \pm 34.7(0-130)$ & $21.4 \pm 30.9(0-127)$ \\
\hline \multicolumn{3}{|c|}{ No. of included perforators (\%) } \\
\hline 1 & $10(41.7)$ & $15(53.6)$ \\
\hline $2<$ & $14(58.3)$ & $13(46.4)$ \\
\hline \multicolumn{3}{|l|}{ Perforator type (\%) } \\
\hline Musculocutaneous & $23(95.8)$ & $26(92.9)$ \\
\hline Septocutaneous & $1(4.2)$ & $2(7.1)$ \\
\hline \multicolumn{3}{|l|}{ Closure method (\%) } \\
\hline Direct closure & $1(4.2)$ & $14(50.0)$ \\
\hline Skin graft & SG: 23 (95.8) & SG: $14(50.0)$ \\
\hline
\end{tabular}

\section{Fig. 2. Delayed healing and additional skin grafting}

(A) Partial loss of grafted skin and fistula under the skin and proximal muscle extension were noted. (B) An incision was made just above the fistula 3 weeks after the initial operation. (C) An additional split-thickness meshed skin graft was applied on the subcutaneous tissue after debridement of the fistula.
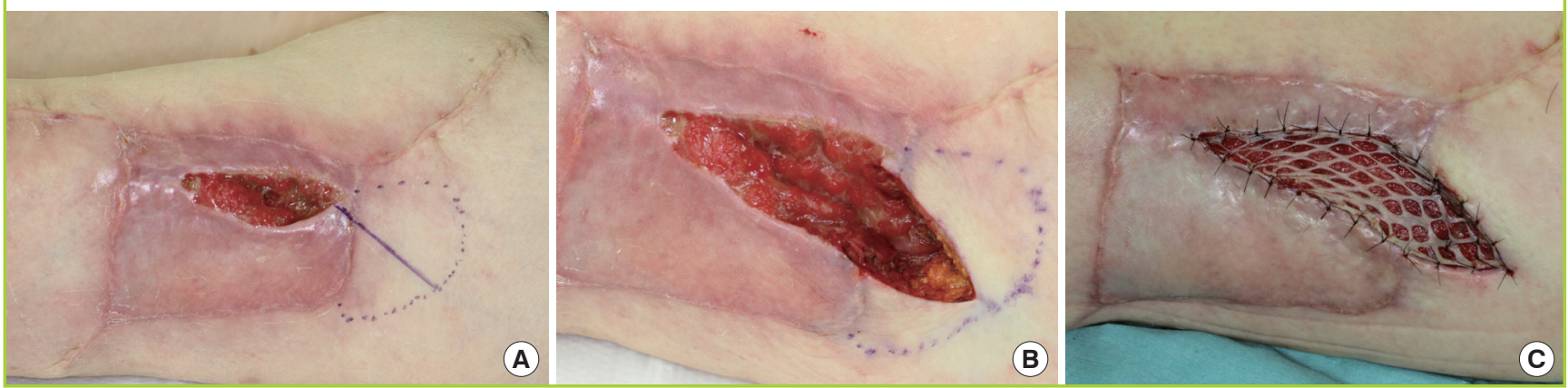

group. The number of patients with a habit of smoking in the delayed healing group was higher than in the normal healing group. In the multivariate logistic regression analysis, the probability of having a habit of smoking was higher in the delayed healing group than in the normal healing group. Among the 52 patients, primary direct closure of the free ALT donor site was performed in 15 patients (29\%) and skin grafting was performed in the remaining 37 patients (71\%). Of the 37 patients who underwent skin grafting, 23 (62\%) experienced delayed healing at the donor site. In contrast, of the 15 patients who underwent primary direct closure, only 1 (7\%) experienced delayed healing at the donor site. Skin grafting was associated with delayed healing at the donor site in multivariate logistic regres- sion analysis.

\section{DISCUSSION}

We could not find previous reports describing the risk factors for delayed healing at the free ALT donor site; however, delayed healing is a severe complication because additional invasive treatment is occasionally required. In the present study, $25 \%$ of patients with delayed healing at the donor site needed additional operations under general anesthesia; moreover, their hospital stay was extended, although normal wound healing was noted at the recipient site.

Using multivariate logistic regression analysis, we found that 
Table 3. Univariate and multivariate logistic regression analysis of risk factors associated with delayed healing at the free ALT flap donor site

\begin{tabular}{|c|c|c|c|c|c|c|}
\hline \multirow{2}{*}{ Variables } & \multicolumn{3}{|c|}{ Univariate logistic regression } & \multicolumn{3}{|c|}{ Multivariate logistic regression } \\
\hline & Odds ratio & $95 \% \mathrm{Cl}$ & P-value & Odds ratio & $95 \% \mathrm{Cl}$ & P-value \\
\hline Age & 0.97 & $0.93-1.01$ & 0.13 & - & - & - \\
\hline Body mass index & 1.42 & $1.14-1.76$ & $0.002^{*}$ & 1.35 & $1.00-1.80$ & $0.044^{\star \star}$ \\
\hline Hypertension & 0.79 & $0.21-2.91$ & 0.72 & - & - & - \\
\hline Hyperlipidemia & 1.06 & $0.33-3.37$ & 0.93 & - & - & - \\
\hline Diabetes mellitus & 2.60 & $0.43-15.65$ & 0.30 & - & - & - \\
\hline Smoking & 5.87 & $1.69-20.36$ & $0.005^{\star}$ & 11.82 & $1.58-88.24$ & $0.016^{\star \star}$ \\
\hline Hemoglobin & 1.40 & $1.01-1.93$ & $0.043^{*}$ & 1.36 & $0.82-2.25$ & 0.23 \\
\hline Total protein & 0.89 & $0.39-2.05$ & 0.79 & - & - & - \\
\hline Albumin & 1.42 & $0.48-4.21$ & 0.53 & - & - & - \\
\hline Skin flap size & 1.01 & $0.99-1.01$ & $0.062^{*}$ & 1.01 & $0.99-1.02$ & 0.20 \\
\hline VLM flap size & 1.01 & $0.99-1.03$ & 0.23 & - & - & - \\
\hline Multiple perforators & 1.62 & $0.54-4.85$ & 0.39 & - & - & - \\
\hline Musculocutaneous perforator & 1.77 & $0.15-20.82$ & 0.65 & - & - & - \\
\hline Skin graft & 23.00 & $2.72-194.47$ & $0.004^{*}$ & 19.49 & $1.24-306.24$ & $0.035^{\star \star}$ \\
\hline
\end{tabular}

the patient factors of a high preoperative BMI and a habit of smoking, as well as the operative factor of skin grafting at the donor site, were risk factors for delayed healing at the free ALT flap donor site. Wound healing is dependent on good nutrition and the presence of suitable polyunsaturated fatty acids in the diet [12]. Protein deficiency has been demonstrated to contribute to poor healing rates, with reduced collagen formation and wound dehiscence [13]. Although various preoperative parameters and nutritional equations have been used to predict postoperative wound healing status, no gold standard methodology exists [14]. No previous reports have explored the correlation between BMI and delayed healing at the free ALT donor site. In a previous prospective study, Baldwin et al. [15] reported a correlation between BMI and delayed wound healing in patients who underwent breast reduction surgery. One of the possible reasons for the association between high BMI and delayed wound healing is the lack of vascularity within abundant fat tissues, resulting in poor skin graft adaptation or revascularization between the subcutaneous tissues and muscles. Another possible reason is the presence of a large amount of exudate, which can prevent adhesion in the subcutaneous tissues or result in seroma formation, even when drain tubes are placed in the subcutaneous spaces.

Many clinicians have reported poor outcomes in smokers. In a review of 758 deep epigastric perforator flaps, Gill et al. [16] reported that the incidence of donor site complications was high in smokers. A previous experimental study described the negative effects of smoking on wound healing [17]. Cigarette smoke contains more than 4,000 toxic compounds, and of these, nico- tine is of the greatest interest. Nicotine has several effects on wound healing. First, it reduces the proliferation of red blood cells, fibroblasts, and macrophages, thereby resulting in decreased tissue oxygenation and subsequent scar formation. Second, it is associated with increased platelet adhesiveness, which can cause microclots and decreased microperfusion [18]. Additionally, nicotine reduces the level of prostacyclin, which is one of the most important physiological vasodilators [19]. Through these mechanisms, nicotine can cause tissue ischemia. Moreover, these mechanisms might affect wound adaptation at the donor site after harvesting of the free ALT flap. These factors would cause a decrease in vascular networks, resulting in delayed wound healing.

In the present study, we found that the incidence of delayed healing was significantly lower in patients who underwent primary closure than in those who underwent skin grafting. The associations of these methods with complications at the donor site are controversial. Some reports stated that primary closure decreased the rate of complications at the ALT flap donor site [20-22], while other reports stated that there was no relationship between complications and the closure method $[7,10,23,24]$. In a large study of 220 patients, Hanasono et al. [24] reported that complications at the free ALT flap donor site were not related to the type of closure, and the authors did not find an increase in the rate of complications associated with skin grafting. However, in that study, $85 \%$ of the patients underwent primary direct closure and only $15 \%$ of the patients underwent skin grafting. Additionally, in a study by Kimata et al. [7], 5 of 37 patients underwent skin grafting, and none of those patients 
showed delayed healing of the ALT donor site. In contrast, in our study, $29 \%$ of the patients underwent primary closure and $71 \%$ of the patients underwent skin grafting. The differences between the findings of our study and those of the studies by Hanasono et al. and Kimata et al. might be related to the differences in the proportion of patients who underwent skin grafting at the donor site.

In our study, over $80 \%$ of the patients underwent reconstruction of head and neck defects using the free ALT flap, and many reconstructions were performed after resection of a relatively large malignant tumor. As described previously [1], we used a large free ALT flap incorporating the VLM for mandibular reconstruction. The harvested ALT and VLM flaps were used to wrap the mandibular reconstruction plate and fill the dead spaces around the plate to prevent plate exposure or refractory ulcers resulting from salivary fistulas. The rate of skin grafting at the donor site was high because large ALT flaps were needed in these cases. Boca et al. [20] reported that BMI and the free ALT flap width-to-thigh circumference ratio were significantly negatively related; therefore, measurement of that ratio provides a more reliable indication of whether the donor site can be closed primarily. An increase in the rate of skin grafting would increase the incidence of delayed wound healing at the free ALT donor site. Procedures for covering the skin defect at the donor site without the use of skin grafting, such as additional pedicled perforator flap surgery [22] and the tissue expansion technique [21], are mainly intended to improve the cosmetic appearance or prevent limitations in the range of motion of the hip and knee due to the contracture that accompanies skin grafting. In addition to those reasons, we recommend that skin grafting should be avoided because of the high rate of delayed wound healing at the free ALT donor site.

One of the disadvantages of the ALT flap has been reported to be variations in vascular anatomy [5]. One possible reason for delayed healing at the donor site is injury of the VLM associated with dissection of perforators in the free ALT flap. The flap perforator may be either a septocutaneous perforator present between the rectus femoris and VLM or a musculocutaneous perforator passing through the VLM. In the case of a musculocutaneous perforator, careful and precise intramuscular dissection with expert techniques is required. After intramuscular dissection or harvesting of the VLM, vascular impairment in the remaining muscle may cause wound dehiscence or unsatisfactory adaptation of the skin graft at the donor site. In the present study, we harvested most of the ALT flaps with musculocutaneous perforators ( $94 \%$; 48 of 51 flaps), with some degree of VLM injury, because septocutaneous perforators were absent. The amount of the VLM incorporated was not significantly associat- ed with delayed wound healing in our study. However, blood flow beneath the skin graft might be insufficient after dissection of the VLM, resulting in partial failure of the skin graft to adapt. The separation of the intermuscular connective tissues between the rectus femoris muscle and vastus intermedius muscle might also be involved. Hematoma or seroma is associated with minute movements, while resting with a molded plastic splint and suction drain tube on the bed for a week might cause wound dehiscence postoperatively. We encountered cases of delayed healing associated with intermuscular fistulae 7-10 days after the operation, despite almost complete adaptation of the skin grafts. Incisions just above the fistulae and additional skin grafts were necessary subsequently. A small seroma or hematoma below an area of unstable adaptation of the skin graft in the early postoperative period could be a cause of a deep fistula after intermuscular separation, and may require additional invasive treatment. However, absorption of this fluid might occur when primary closure is performed. Moreover, aspiration can be performed postoperatively in patients who undergo primary closure when a relatively large seroma is found. This may explain the lower rate of wound dehiscence in the primary closure group compared with the skin graft group. The use of a negative pressure dressing on the grafted skin at the donor site is believed to increase the success rate of adaptation of skin grafts because of the exclusion of redundant fluid in the intermuscular space and the fast immobilization of grafted skin on the muscles [25].

\section{CONFLICT OF INTEREST}

No potential conflict of interest relevant to this article was reported.

\section{PATIENT CONSENT}

The patient provided written informed consent for the publication and the use of their images.

\section{ORCID}

Yoshiro Abe https://orcid.org/0000-0002-7445-2857

\section{REFERENCES}

1. Hashimoto I, Nakanishi H, Yamano M, et al. Usefulness in combined free anterolateral thigh and vastus lateralis muscle flaps. Eur J Plast Surg 2012;35:867-72.

2. Song YG, Chen GZ, Song YL. The free thigh flap: a new free flap concept based on the septocutaneous artery. $\mathrm{Br} \mathrm{J}$ Plast 
Surg 1984;37:149-59.

3. Koshima I, Fukuda H, Yamamoto H, et al. Free anterolateral thigh flaps for reconstruction of head and neck defects. Plast Reconstr Surg 1993;92:421-8.

4. Wei FC, Jain V, Celik N, et al. Have we found an ideal softtissue flap? An experience with 672 anterolateral thigh flaps. Plast Reconstr Surg 2002;109:2219-26.

5. Kuo YR, Jeng SF, Kuo MH, et al. Free anterolateral thigh flap for extremity reconstruction: clinical experience and functional assessment of donor site. Plast Reconstr Surg 2001;107:1766-71.

6. Lakhiani C, Lee MR, Saint-Cyr M. Vascular anatomy of the anterolateral thigh flap: a systematic review. Plast Reconstr Surg 2012;130:1254-68.

7. Kimata Y, Uchiyama K, Ebihara S, et al. Anterolateral thigh flap donor-site complications and morbidity. Plast Reconstr Surg 2000; 106:584-9.

8. Huang $\mathrm{CH}$, Chen $\mathrm{HC}$, Huang YL, et al. Comparison of the radial forearm flap and the thinned anterolateral thigh cutaneous flap for reconstruction of tongue defects: an evaluation of donor-site morbidity. Plast Reconstr Surg 2004;114:170410.

9. Mureau MA, Posch NA, Meeuwis CA, et al. Anterolateral thigh flap reconstruction of large external facial skin defects: a follow-up study on functional and aesthetic recipient- and donor-site outcome. Plast Reconstr Surg 2005;115:107786.

10. Townley WA, Royston EC, Karmiris N, et al. Critical assessment of the anterolateral thigh flap donor site. J Plast Reconstr Aesthet Surg 2011;64:1621-6.

11. Agostini T, Lazzeri D, Spinelli G. Anterolateral thigh flap: systematic literature review of specific donor-site complications and their management. J Craniomaxillofac Surg 2013;41:1521.

12. Albina JE, Gladden P, Walsh WR. Detrimental effects of an omega-3 fatty acid-enriched diet on wound healing. JPEN J Parenter Enteral Nutr 1993;17:519-21.

13. Mistry D, Andrassy RJ, Pizzini R, et al. Quantitative analysis of collagen accumulation during protein malnutrition. J Pe- diatr Surg 1994;29:863-5.

14. Guo JJ, Yang H, Qian H, et al. The effects of different nutritional measurements on delayed wound healing after hip fracture in the elderly.J Surg Res 2010;159:503-8.

15. Baldwin CJ, Kelly EJ, Batchelor AG. The variation in breast density and its relationship to delayed wound healing: a prospective study of 40 reduction mammoplasties. J Plast Reconstr Aesthet Surg 2010;63:663-5.

16. Gill PS, Hunt JP, Guerra AB, et al. A 10-year retrospective review of 758 DIEP flaps for breast reconstruction. Plast Reconstr Surg 2004;113:1153-60.

17. Nolan J, Jenkins RA, Kurihara K, et al. The acute effects of cigarette smoke exposure on experimental skin flaps. Plast Reconstr Surg 1985;75:544-51.

18. van Adrichem LN, Hovius SE, van Strik R, et al. Acute effects of cigarette smoking on microcirculation of the thumb. Br J Plast Surg 1992;45:9-11.

19. Nadler JL, Velasco JS, Horton R. Cigarette smoking inhibits prostacyclin formation. Lancet 1983;1:1248-50.

20. Boca R, Kuo YR, Hsieh CH, et al. A reliable parameter for primary closure of the free anterolateral thigh flap donor site. Plast Reconstr Surg 2010;126:1558-62.

21. Visconti G, Salgarello M. Anteromedial thigh perforator-assisted closure of the anterolateral thigh free flap donor site. J Plast Reconstr Aesthet Surg 2013;66:e189-92.

22. Hallock GG. Tissue expansion techniques to minimize morbidity of the anterolateral thigh perforator flap donor site. J Reconstr Microsurg 2013;29:565-70.

23. Lipa JE, Novak CB, Binhammer PA. Patient-reported donor-site morbidity following anterolateral thigh free flaps. J Reconstr Microsurg 2005;21:365-70.

24. Hanasono MM, Skoracki RJ, Yu P. A prospective study of donor-site morbidity after anterolateral thigh fasciocutaneous and myocutaneous free flap harvest in 220 patients. Plast Reconstr Surg 2010;125:209-14.

25. Moisidis E, Heath T, Boorer C, et al. A prospective, blinded, randomized, controlled clinical trial of topical negative pressure use in skin grafting. Plast Reconstr Surg 2004;114:91722. 